\title{
Lattice Results in Coulomb Gauge
}

\author{
Attilio Cucchieri \\ Instituto de Física de São Carlos, Universidade de São Paulo, Caixa Postal 369, \\ 13560-970 São Carlos, SP, Brazil
}

\begin{abstract}
We discuss recent numerical results obtained for gluon and ghost propagators in lattice Coulomb gauge and the status of the so-called Gribov-Zwanziger confinement scenario in this gauge. Particular emphasis will be given to the eigenvalue spectrum of the Faddeev-Popov matrix.
\end{abstract}

Keywords: Yang-Mills theory; confinement; Green's functions; Gribov copies; Coulomb gauge

PACS: $11.15 . \mathrm{Ha}$ 12.38.Aw

\section{INTRODUCTION}

In recent years several groups have studied confinement of quarks and gluons using lattice simulations in Coulomb gauge [1]-[11]. This gauge has several advantages, even though it breaks the Lorentz symmetry explicitly. Indeed, it is a physical gauge [12], i.e. there are no unphysical degrees of freedom. Also, a nice confinement scenario [13][19] is available for this gauge, based on Gribov's classical work [13]. Finally, Coulomb gauge is well-suited for the Hamiltonian approach and the study of hadron physics by variational methods [20].

Here we review lattice numerical studies in Coulomb gauge. We divide these studies in two periods. In the first period - called here the classical era - the studies focused on the infrared (IR) behavior of propagators (gluon and ghost) [1]-[4] and on the longdistance behavior of the color-Coulomb potential [3]-[8]. On the other hand, in the second period - the modern era - the eigenvalue spectrum of the Faddeev-Popov (FP) operator became the main subject of investigation [9, 10].

\section{COULOMB GAUGE AT THE CLASSICAL LEVEL}

In a classical Yang-Mills theory [12], Gauss's law is written as $\left(D_{i} E_{i}\right)^{a}(\vec{x}, t)=\rho_{q u}^{a}(\vec{x}, t)$, where $D_{i}$ is the gauge-covariant derivative, $E_{i}^{a}(\vec{x}, t)$ is the color-electric field and $\rho_{q u}^{a}(\vec{x}, t)$ is the quark color-charge density. (Here the sum over repeated indices is always understood.) In Coulomb gauge, i.e. $\left(\partial_{i} A_{i}\right)^{a}(\vec{x}, t)=0$, the color-electric field can be decomposed into its transverse and longitudinal parts using $E_{i}^{a}(\vec{x}, t) \equiv\left(E^{t r}\right)_{i}^{a}(\vec{x}, t)-$ $\partial_{i} \phi^{a}(\vec{x}, t)$, where $\phi^{a}(\vec{x}, t)$ is the so-called color-Coulomb potential and $\left(\partial_{i} E_{i}^{t r}\right)^{a}(\vec{x}, t)=$ 0 . Then, Gauss's law becomes $\left(\mathscr{M} \phi^{a}\right)(\vec{x}, t)=\rho^{a}(\vec{x}, t)$, where $\mathscr{M} \equiv-D_{i} \partial_{i}$ is the 3-dimensional FP operator and $\rho^{a}(\vec{x}, t)=\rho_{q}^{a}(\vec{x}, t)-f^{a b c} A_{i}^{b}(\vec{x}, t)\left(E_{i}^{t r}\right)^{c}(\vec{x}, t)$ is the total color-charge density. It follows that the color-Coulomb potential $\phi^{a}(\vec{x}, t)$ can be expressed by means of the instantaneous and non-local operator $\left(\mathscr{M}^{-1}\right)^{a b}(\vec{x}, \vec{y} ; t)$, namely $\phi^{a}(\vec{x}, t)=\left(\mathscr{M}^{-1} \rho\right)^{a}(\vec{x}, t)=\int d^{3} y\left(\mathscr{M}^{-1}\right)^{a b}(\vec{x}, \vec{y} ; t) \rho^{b}(\vec{y}, t)$. At the same time, the 
classical Hamiltonian $\mathscr{H}=\int d^{3} x\left(E_{i}^{2}+B_{i}^{2}\right) / 2$ can be written as $\mathscr{H}=\mathscr{H}_{\text {Coul }}+$ $\int d^{3} x\left[\left(E_{i}^{t r}\right)^{2}+B_{i}^{2}\right] / 2$. Here

$$
\mathscr{H}_{\text {Coul }}=\frac{1}{2} \int d^{3} x\left(\partial_{i} \phi\right)^{2}=\frac{1}{2} \int d^{3} x \int d^{3} y \rho^{a}(\vec{x}) \mathscr{V}^{a b}(\vec{x}, \vec{y}) \rho^{b}(\vec{y}),
$$

$\mathscr{V}^{a b}(\vec{x}, \vec{y})=\left[\mathscr{M}^{-1}(-\Delta) \mathscr{M}^{-1}\right]^{a b}(\vec{x}, \vec{y})$ is the color-Coulomb-potential energy functional and we indicate with $\Delta$ the usual Laplacian.

Clearly, the static color-Coulomb potential $\phi^{a}(\vec{x}, t)$ is closely related to the $3 \mathrm{~d}$ FP (equal-time) ghost propagator $G(\vec{x}-\vec{y}, t) \delta^{a, b} \equiv\left\langle\left(\mathscr{M}^{-1}\right)^{a b}(\vec{x}, \vec{y} ; t)\right\rangle$. In particular, if we consider the Fourier transform $\widetilde{G}(\vec{k}, t)$ we obtain

$$
\phi^{a}(\vec{x}, t) \approx \frac{1}{(2 \pi)^{3}} \int d^{3} k \int d^{3} y \widetilde{G}(\vec{k}, t) \exp \left[i k_{j}\left(x_{j}-y_{j}\right)\right] \rho^{a}(\vec{y}, t) .
$$

Thus, if the ghost propagator has a $k^{-4}=|\vec{k}|^{-4}$ singularity at small momenta we get, in the limit of large separation $x=|\vec{x}|$, a linearly rising potential, i.e. $\phi^{a}(\vec{x}, t) \sim x$.

\section{THE GRIBOV-ZWANZIGER CONFINEMENT SCENARIO}

A non-perturbative investigation of QCD in the IR limit is necessary in order to get an understanding of color confinement. Of course, in developing non-perturbative techniques, one has to deal with the redundant gauge degrees of freedom of the theory. The gauge-fixing technique developed by Faddeev and Popov assumed that one could find a gauge-fixing condition that uniquely determines a gauge field on each gauge orbit. However, in Ref. [13] Gribov showed that the Coulomb and the Landau gauge conditions do not fix the gauge fields uniquely, namely there exist gauge-related field configurations that satisfy the gauge condition (Gribov copies) [13, 19].

In order to get rid of the problem of spurious gauge copies, Gribov proposed the use of additional gauge conditions. In particular, for Coulomb gauge, he proposed the restriction of the physical configuration space (on each time-slice $t$ ) to the region $\Omega_{t} \equiv$ $\left\{A:\left(\partial_{i} A_{i}\right)^{a}(\vec{x}, t)=0, \mathscr{M}^{a b}(\vec{x}, \vec{y} ; t) \geq 0\right\}$. Thus, inside the region $\Omega_{t}$, the FP operator has no negative eigenvalues. This region is delimited by the so-called first Gribov horizon $\partial \Omega_{t}$, where the smallest non-trivial eigenvalue of the FP operator $\mathscr{M}^{a b}(\vec{x}, \vec{y} ; t)$ is zero. On the lattice, given a thermalized lattice configuration $\{U(x)\}$, a configuration belonging to the region $\Omega_{t}$ can be obtained by finding a gauge transformation $\{g(x)\}$ that brings the functional $1 \mathscr{E}_{\mathrm{hor}, U}[g]=-\sum_{i=1}^{3} \sum_{\vec{x}, t} \operatorname{Tr}\left[g(\vec{x}, t) U_{i}(\vec{x}, t) g^{\dagger}\left(\vec{x}+a e_{i}, t\right)\right]$ to a local minimum. Recall that, in the $S U\left(N_{c}\right)$ case, both the link variables $U_{\mu}(x)$ and the gauge transformation matrices $g(x)$ are elements of the $S U\left(N_{c}\right)$ group (in the fundamental $N_{c} \times N_{c}$ representation).

\footnotetext{
${ }^{1}$ In this review we do not discuss results related to the (possible) spontaneous symmetry breaking of the residual gauge freedom $g(t)[6,7,11]$ and to the so-called $\lambda$ gauge [21], which interpolates between the Landau gauge $(\lambda=1)$ and a Coulomb-gauge like condition $(\lambda \rightarrow 0)$.
} 
The additional gauge condition added by Gribov is not significant for the highfrequency vacuum fluctuations, i.e. for the perturbative regime, but it suppresses the low-frequency fluctuations, modifying the (non-perturbative) IR regime [13, 14]. In particular, one can show that, when the functional integration is restricted to the region $\Omega_{t}$, then (on each time slice) the ghost propagator $G(\vec{k}, t)$ is IR enhanced. On the other hand, the transverse gluon propagator $D^{t r}(\vec{k}, t)$ may go to zero in the IR limit, implying a maximal violation of reflection positivity. The latter result may be viewed as an indication of gluon confinement [22]. Analytic results for the IR behavior of propagators and vertices using Dyson-Schwinger equations have been presented in Ref. [23].

At the same time, the 44-component of the gluon propagator can be written [15] as $D_{44}(\vec{x}-\vec{y}, t)=V_{\text {Coul }}(\vec{x}-\vec{y}) \delta(t)+P(\vec{x}-\vec{y}, t)$, where $V_{\text {Coul }}(\vec{x}-\vec{y}) \delta^{a b}=\left\langle\mathscr{V}^{a b}(\vec{x}, \vec{y})\right\rangle$ is anti-screening and should yield a linearly rising potential, while $P(\vec{x}-\vec{y}, t)$ is the vacuum-polarization term, i.e. it is responsible for screening and for the breaking of the string between color sources. One can show that these three quantities [e.g. $D_{44}(\vec{x}-\vec{y}, t)$, $V_{\text {Coul }}(\vec{x}-\vec{y})$ and $\left.P(\vec{x}-\vec{y}, t)\right]$ are renormalization-group invariant [15, 16]. One can also define the running coupling constant

$$
g_{\text {Coul }}^{2}(\vec{k})=\frac{11 N_{c}-2 N_{f}}{12 N_{c}} k^{2} V_{\text {Coul }}(\vec{k})
$$

Clearly, if the color-Coulomb potential $V_{\text {Coul }}(x)$ is linearly rising at large separation $x$, then in the IR limit we find $V_{\text {Coul }}(\vec{k}) \sim 1 / k^{4}$ and $g_{\text {Coul }}^{2}(\vec{k}) \sim 1 / k^{2}$. Also, it has been shown [17] that the Coulomb energy of static sources is an upper bound for the static inter-quark potential $V(\vec{x})$, i.e. if at large $x$ one has $V_{\text {Coul }}(\vec{x})=\sigma_{\text {Coul }} x$ and $V(\vec{x})=\sigma x$ then we find $\left(N_{c}^{2}-1\right) \sigma_{\text {Coul }} /\left(2 N_{c}\right) \geq \sigma$. Analytic results for the long distance behavior of $V_{\text {Coul }}(\vec{x})$ have been presented in Ref. [18].

Summarizing [24], in the Gribov-Zwanziger confinement scenario (in Coulomb gauge), the long-range force, responsible for color confinement, is carried by an instantaneous static color-Coulomb field. In particular, the linearly rising potential is related to the IR divergence of the ghost-propagator (at equal time). At the same time, the propagator of three-dimensionally transverse (would-be physical) gluons is IR suppressed and the gluons are absent from the spectrum.

\section{THE CLASSICAL ERA: RESULTS}

The analytic predictions described above for the gluon propagators $D^{\operatorname{tr}}(\vec{k})$ and $D_{44}(\vec{k})$ have been verified for the $S U(2)$ group in Refs. [1]-[4]. In particular, from Fig. 1 of Ref. [1] it is evident that, in the IR limit, the transverse propagator is suppressed, while $D_{44}(\vec{k})$ blows up. Moreover, in the infinite-volume limit, it has been found [1, 2] that $D^{\operatorname{tr}}(\vec{k})$ is well described a Gribov-like propagator characterized by a pair of purely imaginary poles $m^{2}= \pm i y$. Numerically, at $\beta=2.2$ and in the infinite-volume limit, one finds $y=0.33 \pm 0.14 \mathrm{GeV}^{2}$. As for the ghost propagator $G(\vec{k}, t)$, it has been studied up to now only in Ref. [4]. There, it is shown that $G(\vec{k}, t)$ has indeed an IR divergence stronger than $1 / k^{2}$. At the same time, the running coupling $g_{\text {Coul }}^{2}(\vec{k})$, defined in Eq. (3) above, seems 
to be consistent [3, 4] with an IR behavior of the type $1 / k^{2}$. These analyses have also obtained $\sigma_{\text {Coul }} \approx \sigma$.

In Ref. [5], the color-Coulomb potential $V_{\text {Coul }}(\vec{x})$ has been evaluated [for the $S U(2)$ group $]^{2}$ as a function of the separation $x$, using correlators of two time-like Wilson lines of length 1 (in lattice units). It was found that $V_{\text {Coul }}(\vec{x})$ increases linearly with $x$, in agreement with the $1 / k^{2}$ behavior for $g_{\text {Coul }}^{2}(\vec{k})$ obtained in Refs. [3, 4]. However, in this case the estimate for the Coulomb string tension was $\sigma_{\text {Coul }} \approx 2-3 \sigma$. Moreover, if one removes the so-called center vortices [5], then the color-Coulomb potential $V_{\text {Coul }}(\vec{x})$ goes to a constant at large $x$ and $\sigma_{\text {Coul }}=0$. This suggests a strong relation between these center vortices and the (Coulomb) confinement mechanism. Note that similar effects have been observed in the gluon and in the ghost propagators in Landau gauge [25] after removing the center vortices.

It is also interesting that, when the temperature is turned on [7, 8], the color-Coulomb potential $V_{\text {Coul }}(\vec{x})$ is not screened and it is still a linearly rising function of $x$. Moreover, the Coulomb string tension $\sigma_{\text {Coul }}$ shows a magnetic-like behavior [8], i.e. $\sigma_{\text {Coul }}^{1 / 2} \sim$ $g^{2}(T) T$. This implies that the Coulomb string tension cannot be used as an order parameter for confinement. This conclusion can be understood by observing that the temperature is defined by compactifying the time direction and that the Coulomb gauge is defined on the subspace orthogonal to the time direction. Thus, there is no reason for the system in Coulomb gauge to be sensitive to the deconfining transition.

\section{THE MODERN ERA}

In Ref. [9] the authors evaluate the gauge-field excitation energy $\mathscr{E}$ (above the ground state energy) of a single (point-like) static color charge in Coulomb gauge. Considering that long-range effects should be related to the non-local-interaction term $\mathscr{H}_{\text {Coul }}$, one finds

$$
\mathscr{E} \propto \mathscr{V}^{a a}(x, x)=\left[\mathscr{M}^{-1}(-\Delta) \mathscr{M}^{-1}\right]^{a a}(x, x) .
$$

A necessary condition for confinement is that $\mathscr{E}$ should diverge in the infinite-volume limit, due to IR effects. (Ultraviolet divergences are regulated by the lattice cut-off.) For the (Coulomb) FP matrix $\mathscr{M}^{a b}=-\delta^{a b} \Delta-f^{a c b} A_{\mu}^{c} \partial_{\mu}$ one can consider (inside the Gribov region $\Omega_{t}$ ) the eigenvalues $\lambda>0$ and the corresponding eigenfunctions $\Phi_{\lambda, x}^{a}$. Then, Eq. (4) can be written as $\mathscr{E} \propto\left\langle\sum_{\lambda} F_{\lambda} / \lambda^{2}\right\rangle$ with $F_{\lambda}=V_{s}^{-1} \sum_{x y}\left(\Phi_{\lambda, x}^{a}\right)^{*}(-\Delta)_{x, y}\left(\Phi_{\lambda, y}^{a}\right)$. (Here, $V_{s}$ is the $3 \mathrm{~d}$ spatial volume of the lattice.) For a sufficiently large volume, the sums can be approximated by integrals and $\mathscr{E} \propto\left\langle\int_{\lambda_{\min }}^{\lambda_{\max }} d \lambda \rho(\lambda) F(\lambda) / \lambda^{2}\right\rangle$, with $\int d \lambda \rho(\lambda)=1$. In the infinite-volume limit, the volume of the Gribov region gets concentrated near the Gribov horizon $[9,17,26]$, i.e. $\lambda_{\min } \rightarrow 0$. In the same limit, the gaugefield excitation energy $\mathscr{E}$ blows up if

$$
\lim _{\lambda \rightarrow 0} \frac{\rho(\lambda) F(\lambda)}{\lambda}>0 .
$$

${ }^{2}$ Similar results were obtained for the $S U(3)$ group in Refs. [7, 8]. 
Thus, a necessary condition for confinement is the enhancement of $\rho(\lambda) F(\lambda)$ at small momenta.

In Appendix A of Ref. [9], an interesting analysis based on a random-matrix model shows that, for small eigenvalues, one should have $\rho(\lambda)=c \lambda^{\alpha}$ if the eigenvalues $\lambda$ scale as $V_{s}^{-1 /(1+\alpha)}$ if the volume is increased. Numerically they find $\alpha=0.25(5)$, implying $\lambda_{\text {min }} \sim 1 / L^{2.4}$. At the same time, they obtain $F\left(\lambda_{\text {min }}\right) \sim 1 / L \sim \lambda_{\text {min }}^{0.38}$ and the confinement criterion (5) is clearly fulfilled [9, 10]. A similar result is obtained when considering the so-called "vortex-only" configurations [9]. On the other hand, after removing the center vortices, one recovers a Laplacian-like eigenvalue spectrum for the FP operator $\mathscr{M}_{x y}^{a b}$ with $\lim _{\lambda \rightarrow 0} \rho(\lambda) F(\lambda) / \lambda=0$. Thus, in agreement with the findings reported in the previous Section, the enhancement of $\rho(\lambda) F(\lambda)$ at small eigenvalues $\lambda$ and the confinement mechanism in Coulomb gauge seem to be strictly related to the properties of the center-vortex configurations. One can also show [9] that center-vortex configurations are (infinitely many) distinguished points on the Gribov horizon. The relation between these configurations and the Gribov-Zwanziger scenario in Coulomb gauge is then clear if, in the infinite-volume limit, the center-vortex configurations are sufficiently dense on the Gribov horizon.

\section{AN OPEN QUESTION FOR THE FUTURE}

In order to understand fully the Gribov-Zwanziger confinement scenario one should consider a generic gauge condition $\mathscr{F}[A]=0$, imposed by minimizing a functional $E[U]$. Then, from the second variation of $E[U]$, we can always define the FP matrix $\mathscr{M}_{x y}^{a b}$. Clearly, when we are at a (local) minimum of $E[U]$, the (non-trivial) eigenvalues of $\mathscr{M}_{x y}^{a b}$ are positive and we can define a Gribov region $\Omega$ and the first Gribov horizon $\partial \Omega$. Moreover, since the configuration space has a very large dimensionality, entropy should favor (in the limit of large volumes) configurations near the Gribov horizon [9, 17, 26], i.e. $\lambda_{\min }$ should go to zero in the same limit. This is indeed the case in $3 \mathrm{~d}$ [27] and 4d Landau gauge [28], in 4d Coulomb gauge [9] and in 4d Maximally Abelian gauge (MAG) [29]. Since the FP matrix develops a null eigenvalue at the Gribov horizon $\partial \Omega$, we should also expect the corresponding ghost propagator $G(k)$ to blow up at small momenta in the infinite-volume limit. This result should in turn introduce a long-range effect in the theory, being probably related to the color-confinement mechanism. Indeed, we know from several numerical studies that the ghost propagator $G(k)$ is IR enhanced in $3 d$ [27] and $4 d$ Landau gauge [25, 30, 31] and in 4d Coulomb gauge [4]. On the other hand, recent numerical results in MAG [29] suggest an IR finite $G(k)$. Thus, the line of thinking reported above cannot be completely correct. Of course, one does not expect the ghost propagator to be particularly important for confinement in MAG, since in this case the accepted scenario is that confinement is related to Abelian dominance and (therefore) to the IR behavior of the diagonal gluon propagator [29, 32]. In any case, we should try to answer the following question: what makes the ghost propagator IR enhanced in Coulomb and in Landau gauge but IR finite in MAG?

A possible solution comes from the observation that in Landau [27, 28] and in Coulomb gauge [9] $\lambda_{\min } \sim 1 / L^{2+\alpha}$ with $\alpha>0$, i.e. it goes to zero faster than in the case 
of the Laplacian. On the contrary, in MAG [29] one has $\lambda_{\min } \sim 1 / L^{2-\alpha}$ (with $\alpha>0$ ), i.e. it goes to zero more slowly than for the Laplacian. This (unproven) hypothesis seems to be supported by the following observation. Using the same notation introduced in the previous section and in the limit of a large volume, we can write the ghost propagator $G(k)$ as

$$
G(k)=\int_{\lambda_{\min }}^{\lambda_{\max }} d \lambda \frac{\rho(\lambda) f_{\lambda}(k)}{\lambda}, \quad f_{\lambda}(k)=\frac{1}{N_{c}^{2}-1} \sum_{a}\left|\Phi_{\lambda}^{a}(k)\right|^{2} .
$$

If we consider a FP matrix of the type $\mathscr{M}^{a b}=-\delta^{a b} \Delta-K^{a b}$ (this is the case in Landau, Coulomb and MAG) then we have

$$
G(k)=\int_{\lambda_{\min }}^{\lambda_{\max }} \frac{d \lambda}{\lambda} \rho(\lambda) \frac{1}{N_{c}^{2}-1} \sum_{a}\left|\Phi_{\lambda}^{a}(k)\right|^{2}, \quad \Phi_{\lambda}^{a}(k)=\frac{1}{k^{2}-\lambda} \sum_{x, y} e^{-i k x} K_{x y}^{a b} \Phi_{\lambda, y}^{b} .
$$

In a numerical simulation we look at $G\left(k_{\text {min }}\right)$ when the volume increases (and $\lambda_{\text {min }}$ decreases). Thus, the IR behavior of $G\left(k_{\min }\right)$ depends on the quantity

$$
\Phi_{\lambda_{\min }}^{a}\left(k_{\min }\right)=\frac{1}{k_{\min }^{2}-\lambda_{\min }} \sum_{x, y} e^{-i k_{\min } x} K_{x y}^{a b} \Phi_{\lambda_{\min }, y}^{b}
$$

and there is a clear competition between the smallest eigenvalue of the Laplacian $k_{\min }^{2} \sim$ $L^{-2}$ and the smallest eigenvalue of the FP operator $\lambda_{\text {min }}$.

It is interesting to notice that using Eq. (6) we can easily explain why finite-size effects are small ${ }^{3}$ when the ghost propagator $G(k)$ is evaluated numerically. Indeed, it is sufficient to have $\rho(\lambda) f_{\lambda}(k) / \lambda \sim \lambda^{\beta}$ with $\beta>-1$ in the limit of small eigenvalues $\lambda$. In Ref. [28] it has been obtained (for 4d Landau gauge) that the quantity $R(\bar{\lambda})=$ $\int_{\lambda_{\min }}^{\bar{\lambda}} d \lambda \rho(\lambda) f_{\lambda}(k) \lambda^{-1} / G(k)$ behaves as $\bar{\lambda}^{v}$, with $v>0$, for small $\bar{\lambda}$ considering the two smallest nonzero momenta $k$. This implies $\beta=v-1>-1$.

\section{CONCLUSIONS}

We believe that the study of the spectral properties of the FP operator in different gauges can help us understand the general features of the Gribov-Zwanziger confinement scenario. In particular, it would be important to clarify for which gauge conditions the confinement mechanism can be related to an enhancement of the ghost propagator in the IR limit.

\footnotetext{
3 Note that in Ref. [31] there are, actually, strong finite-size effects for the ghost propagator $G(k)$. However, in that case the effects are probably due to the use of strong asymmetric lattices, with different ratios of the spatial over the temporal extension of the lattice.
} 


\section{ACKNOWLEDGMENTS}

The author thanks the organizers for the invitation to present this review at QCHS7 and A. Maas, T. Mendes and D. Zwanziger for helpful discussions. This work was partially supported by FAPESP (under grants \# 00/05047-5 and 06/57316-6) and by CNPq.

\section{REFERENCES}

1. A. Cucchieri and D. Zwanziger, Phys. Rev. D 65, 014001 (2002).

2. A. Cucchieri and D. Zwanziger, Phys. Lett. B 524, 123 (2002); A. Cucchieri, T. Mendes and D. Zwanziger, Nucl. Phys. Proc. Suppl. 106, 697 (2002).

3. A. Cucchieri and D. Zwanziger, Nucl. Phys. Proc. Suppl. 119, 727 (2003).

4. K. Langfeld and L. Moyaerts, Phys. Rev. D 70, 074507 (2004).

5. J. Greensite and S. Olejnik, Phys. Rev. D 67, 094503 (2003).

6. J. Greensite, S. Olejnik and D. Zwanziger, Phys. Rev. D 69, 074506 (2004).

7. A. Nakamura and T. Saito, Prog. Theor. Phys. 115, 189 (2006).

8. Y. Nakagawa et al., Phys. Rev. D 73, 094504 (2006).

9. J. Greensite, S. Olejnik and D. Zwanziger, JHEP 0505, 070 (2005).

10. Y. Nakagawa et al., hep-lat/0610128.

11. M. Grady, hep-lat/0607013; M. Grady, hep-lat/0610042

12. See for example Particle Physics and Introduction to Field Theory, T. D. Lee (Harwood Academic Publishers, New York, 1981).

13. V. N. Gribov, Nucl. Phys. B 139, 1 (1978).

14. D. Zwanziger, Phys. Lett. B 257, 168 (1991); D. Zwanziger, Nucl. Phys. B 364 (1991) 127.

15. D. Zwanziger, Nucl. Phys. B 518 (1998) 237; A. Cucchieri and D. Zwanziger, Phys. Rev. D 65, 014002 (2002).

16. A. Cucchieri and D. Zwanziger, Nucl. Phys. Proc. Suppl. 106, 694 (2002).

17. D. Zwanziger, Phys. Rev. Lett. 90, 102001 (2003).

18. D. Zwanziger, Phys. Rev. D 70, 094034 (2004).

19. Y. L. Dokshitzer and D. E. Kharzeev, Ann. Rev. Nucl. Part. Sci. 54, 487 (2004); R. F. Sobreiro and S. P. Sorella, hep-th/0504095.

20. D. Zwanziger, Nucl. Phys. B 485, 185 (1997); D. Zwanziger, hep-th/9710157; A. Cucchieri and D. Zwanziger, Nucl. Phys. Proc. Suppl. 53, 815 (1997); A. Cucchieri and D. Zwanziger, Phys. Rev. Lett. 78, 3814 (1997); A. P. Szczepaniak and E. S. Swanson, Phys. Rev. D 65, 025012 (2002); A. P. Szczepaniak, Phys. Rev. D 69, 074031 (2004); C. Feuchter and H. Reinhardt, Phys. Rev. D 70, 105021 (2004); S. M. Antunes et al., Braz. J. Phys. 35 (2005) 877; A. P. Szczepaniak and P. Krupinski, Phys. Rev. D 73, 034022 (2006); A. P. Szczepaniak and P. Krupinski, Phys. Rev. D 73, 116002 (2006).

21. L. Baulieu and D. Zwanziger, Nucl. Phys. B 548, 527 (1999); A. Cucchieri and T. Mendes, hep-lat/9902024; C. S. Fischer and D. Zwanziger, Phys. Rev. D 72, 054005 (2005); A. Maas, A. Cucchieri and T. Mendes, arXiv:hep-lat/0610123

22. R. Alkofer and L. von Smekal, Phys. Rept. 353, 281 (2001).

23. W. Schleifenbaum, M. Leder and H. Reinhardt, Phys. Rev. D 73, 125019 (2006).

24. See for example D. Zwanziger, hep-ph/0610021.

25. J. Gattnar, K. Langfeld and H. Reinhardt, Phys. Rev. Lett. 93, 061601 (2004).

26. D. Zwanziger, Nucl. Phys. B 412, 657 (1994); A. Cucchieri, Nucl. Phys. B 521, 365 (1998).

27. A. Cucchieri, A. Maas and T. Mendes, Phys. Rev. D 74, 014503 (2006).

28. A. Sternbeck, E. M. Ilgenfritz and M. Muller-Preussker, Phys. Rev. D 73, 014502 (2006).

29. T. Mendes, A. Cucchieri and A. Mihara, hep-lat/0611002.

30. A. Cucchieri, Nucl. Phys. B 508, 353 (1997); S. Furui and H. Nakajima, Phys. Rev. D 69, 074505 (2004); J. C. R. Bloch et al., Nucl. Phys. B 687, 76 (2004); A. Sternbeck et al., Phys. Rev. D 72 , 014507 (2005); A. Cucchieri and T. Mendes, Phys. Rev. D 73, 071502 (2006).

31. O. Oliveira and P. J. Silva, arXiv:hep-lat/0609036

32. Z. F. Ezawa and A. Iwazaki, Phys. Rev. D 25, 2681 (1982); K. Amemiya and H. Suganuma, Phys. Rev. D 60, 114509 (1999); V. G. Bornyakov et al., Phys. Lett. B 559, 214 (2003). 